\title{
COMPLICACIONES DE LA URETERO-RENOSCOPIA
}

\author{
J.M. ALAPONT ALACREU, E. BROSETA RICO, J.L. PONTONES MORENO, \\ F. OLIVER AMORÓS, J.L. PALMERO MARTÍ, F. BORONAT TORMO, \\ J.F. JIMÉNEZ CRUZ
}

Servicio de Urología. Hospital Universitario La Fe. Valencia.

Actas Urol Esp. 27 (9): 692-699, 2003

\section{RESUMEN}

COMPLICACIONES DE LA URETERO-RENOSCOPIA

INTRODUCCIÓN: Desde que en 1980 se introdujera la ureteroscopia (URS) en la práctica urológica los grandes avances tecnológicos han permitido que esta técnica se convierta en un instrumento bastante seguro y eficaz para el diagnóstico y tratamiento de la patología del tracto urinario superior. Sin embargo es una técnica invasiva y por lo tanto no está exenta de riesgos, pudiendo ocasionar en algunas ocasiones complicaciones graves.

MATERIAL Y MÉTODOS: Entre enero de 1990 y diciembre de 2001 hemos realizado 4.645 URS, 2.972 (64\%) en mujeres y 1.673 (36\%) en hombres, con edades comprendidas entre 9 y 82 años (media 49,8). Del total 2.508 $(53,9 \%)$ se hicieron con sedación y $2.137(46,1 \%)$ con anestesia (general o raquídea). Los objetivos de las URS han sido tanto diagnósticos (hematurias de origen desconocido) como terapéuticos (cálculos ureterales, tumores del tracto urinario superior, estenosis ureterales y colocaciones de catéteres ureterales).

RESULTADOS: La complicación más frecuente ha sido la fiebre $(11,7 \%)$, evolucionando a sepsis en 15 pacientes. El resto de complicaciones fueron: perforación ureteral $(1,2 \%)$, avulsión ureteral $(0,06 \%)$, rotura renal $(0,04 \%)$, extrusión litiásica $(0,4 \%)$ y fístula uretero-ilíaca $(0,02 \%)$. La mayoría se resolvieron con tratamiento conservador y en muchos casos endoscópico.

CONCLUSIONES: La URS ha supuesto un gran avance en la urología, tanto con fines diagnósticos como terapéuticos. Cuidadosamente realizada y bien indicada es una técnica segura, eficaz y con escasa morbilidad, siendo practicada en muchos casos sin necesidad de anestesia. En la mayoría de las ocasiones las complicaciones se pueden resolver de manera conservadora y muchas endoscópicamente.

PALABRAS CLAVE: Ureteroscopia. Complicaciones. Litiasis.

\section{ABSTRACT}

URETERO-RENOSCOPY COMPLICATIONS

INTRODUCTION: Since its clinical introduction ureteroscopy (URS) has experienced an impressive development due to the technical improvements of new and smaller urological armamentarium. Currently, ureteroscopy is a worldwide procedure with a varied number of diagnostic and therapeutic possibilities. However, the technique has complications.

MATERIAL AND METHODS: We analyse the complications of URS in a series of 4.645 ureteroscopic procedures performed from january 1990 to december 2001, 2972 (64\%) female and 1673 (36) male. The objectives of URS were diagnostic (haematuria) and therapeutic (ureteral stones, tumors, strictures and placement of stents).

RESULTS: Fever was the most frequent complication (11.7\%), with sepsis in 15 patients. Another complications were: ureteral perforation (1.2\%), ureteral avulsion $(0.06 \%)$, renal injury $(0.04 \%)$, extrusion $(0.4 \%)$ and uretero-iliac fistulae $(0.02 \%)$. The most common treatment was conservative with endourological approach.

CONCLUSION: Carefully performed ureteroscopy is a superb tool for the urologist either for diagnostic or therapeutic purposes with a low ratio of complications. The majority of these complications can be solved with conservative management.

KEY WORDS: Ureteroscopy. Complications. Lithiasis. 
$\mathrm{D}^{\mathrm{s}}$ esde que en 1980 Pérez-Castro y MartínezPiñeiro introdujeran por primera vez la uretero-renoscopia (URS) en la práctica urológica los grandes avances tecnológicos y la aparición de ureteroscopios de mínimo calibre han permitido que esta técnica se convierta en un instrumento seguro y eficaz para el diagnóstico y tratamiento de la patología del tracto urinario superior (litiasis, tumores uroteliales, estenosis, divertículos caliciales, malformaciones vasculares, colocación y retirada de catéteres ureterales y diagnóstico de defectos de repleción o hematurias de origen desconocido), siendo su indicación más común el tratamiento de la litiasis ureteral.

Sin embargo la URS es una técnica invasiva y no está exenta de riesgos, pudiendo provocar hematuria, sepsis, perforación ureteral, urinoma, estenosis ureteral, extrusión litiásica paraureteral y menos frecuentemente rotura renal y avulsión ureteral. Presentamos nuestra experiencia en el diagnóstico y tratamiento de las complicaciones derivadas de las primeras 4.645 URS realizadas en nuestra unidad.

\section{MATERIAL Y MÉTODOS}

En el periodo comprendido entre enero de 1990 y diciembre de 2001 se han realizado en la unidad de litotricia de nuestro hospital un total de 4.645 procedimientos ureteroscópicos. De ellas 2.972 (64\%) eran mujeres y 1.673 (36\%) hombres, con edades comprendidas entre 9 y 82 años (media 49,8).

Del total $2.508(53,9 \%)$ fueron realizadas con sedación (meperidina y remifentanilo intravenoso) y $2.137(46,1 \%)$ casos con raquianestesia o anestesia general. En todos los pacientes realizamos profilaxis antibiótica con una dosis de 100 mg de tobramicina previa al tratamiento y una segunda dosis 8 horas después.

Los ureteroscopios utilizados durante este periodo han sido todos semiflexibles (ACMI, Olympus, Candela y Storz), con calibres entre 7,2 y 11,5 Ch. Durante la ureteroscopia mantenemos una irrigación continua con suero salino por medio de una bomba de perfusión, que nos permite graduar el flujo/presión requerido en cada momento de la instrumentación. No usamos gravedad porque este sistema de perfusión presenta el inconveniente del escaso flujo que obtenemos durante la ocupación del canal de trabajo con pinzas o guías, lo cual comporta una falta de adecuada visión. Es por ello que preferimos la irrigación mediante bomba.

En 282 intervenciones no se consiguió acceder al uréter por diversos motivos: imposibilidad de localizar el meato ureteral o situación inaccesible de éste por reimplante previo, estenosis del meato imposible de dilatar y angulación ureteral excesiva en pacientes con adenomas prostáticos de gran tamaño. La mayoría de URS en las que sí se consiguió acceder al uréter han sido para tratamiento de litiasis. Así se han tratado un total de 4.082 cálculos, 2.664 (65,3\%) en mujeres y $1.418(34,7 \%)$ en hombres, a los que se agrupó en 2 categorías: primarios (no tratados o no modificados radiológicamente por tratamiento previo con litotricia extracorpórea por ondas de choque-LEOC-), correspondiendo a este grupo 2.178 cálculos $(53,3 \%, 1.861$ en mujeres y 317 en hombres); y secundarios (modificados por tratamiento previo con LEOC, calles litiásicas post-LEOC y restos litiásicos persistentes tras URS): 1.904 litiasis $(46,7 \%$, 803 en mujeres y 1.101 en hombres), de las cuales 272 eran calles litiásicas (189 mujeres y 83 hombres). Radiológicamente se establecen 3 niveles en relación a la localización de la litiasis: pelviana, la situada por debajo del reborde de la articulación sacroilíaca, 2.652 casos (43 bilaterales); ilíaca, superpuesta a la articulación sacroilíaca, 1.020 casos; y lumbar, por encima de dicha articulación, 367 casos. La mayoría de los pacientes con litiasis han sido tratados con láser colorante (Candela-MUD) y fibra de $320 \mu$. En 36 pacientes, bien porque el cálculo era muy grande o por mal funcionamiento del láser, se realizaron los tratamientos con lithoclast. Los resultados terapéuticos se valoran mediante radioscopia peroperatoria, radiografía simple de aparato urinario, urografía intravenosa y ecografía. Realizamos un seguimiento mínimo del paciente de 3 meses, con un primer control con radiografía simple y ecografía renal al mes de tratamiento y un segundo con urografía intravenosa (UIV) al tercer mes post-tratamiento y eventualmente sólo con ecografía si es alérgico al contraste o tenemos la seguridad de haber extraido el cálculo en su totalidad. 
Se realizaron 36 URS para tratamiento conservador de tumores del tracto urinario superior $(7 \mathrm{de}$ localización pielo-calicial y 29 a nivel ureteral) efectuando biopsia con pinzas y electrocoagulación de la base tumoral. Si el tumor era de tamaño no tratable por vía endoscópica, sólo se tomaba la biopsia y se programaba la resolución por cirugía abierta.

En 32 casos el objetivo de la URS fue el diagnóstico de hematurias de origen desconocido, en 30 la resolución de estenosis ureterales y en 183 la colocación de catéteres doble $\mathrm{J}$ ureterales de difícil emplazamiento con los métodos convencionales.

\section{RESULTADOS}

La fiebre en el post-operatorio inmediato ha sido la complicación más frecuente tras la URS (Tabla I). Del total, $15(0,32 \%)$ desarrollaron un proceso séptico que se trató con antibioterapia de amplio espectro y derivación urinaria con catéter doble $\mathrm{J}$, evolucionando todos los pacientes de forma satisfactoria excepto 2 que fallecieron por complicaciones en la sepsis.

Tuvimos 56 casos $(1,2 \%)$ de perforación ureteral. Esta fue grave porque precisó suspender el tratamiento o requirió de maniobras terapéuticas posteriores en 13 pacientes. De ellos 7 se detectaron durante el propio acto terapéutico y los otros 6 mediante estudios diagnósticos complementarios (en uno de ellos mediante UIV en el curso de un cuadro de abdomen agudo a las 24 horas de la intervención); la resolución de esta complicación se consiguió mediante la colocación de un catéter doble $\mathrm{J}$ en 10 pacientes, nefrostomía percutánea en dos y otro precisó ureterolitotomía. En 3 casos se ha constatado una estenosis ureteral secundaria.

\section{TABLA I}

\begin{tabular}{|l|c|c|}
\hline Complicación & № & \% \\
\hline Fiebre $>38^{\circ}$ C/Sepsis & $546 / 15$ & $11,7 / 0,32$ \\
\hline Perforación ureteral & 56 & 1,2 \\
\hline Avulsión ureteral & 3 & 0,06 \\
\hline Rotura renal & 2 & 0,04 \\
\hline Extrusión litiásica & 19 & 0,4 \\
\hline Fístula uretero-iliaca & 1 & 0,02 \\
\hline Total & 642 & 13,74 \\
\hline
\end{tabular}

En 3 pacientes $(0,06 \%)$ se produjo una avulsión ureteral. Los 2 primeros casos sucedieron tras extracción de una litiasis localizada a nivel de uréter lumbar con una cesta de Dormia ${ }^{\circledR}$; en uno se realizó una ureteroneocistostomía con colgajo de Boari (Fig. 1) y en el otro, dado que el riñón afecto tenía un importante déficit funcional, una nefrectomía inmediata. El tercer caso ocurrió en un paciente con litiasis en uréter ilíaco derecho en el que tras finalizar la litofragmentación y retirar el ureterorrenoscopio, éste arrancó la mucosa ureteral que quedó evertida unos 5 $\mathrm{cm}$ en la vejiga; la extensión de la avulsión fue limitada por lo que decidimos intentar resituar la mucosa ureteral dentro del meato con la ayuda de un catéter doble $\mathrm{J}$ de mayor calibre, el cual introdujimos sobre la guía de seguridad que habíamos colocado al principio de la intervención, logrando el objetivo. A los 3 meses se retiró el catéter, quedando una estenosis a nivel del uréter distal que se resolvió mediante dilatación neumática endoscópica. Posteriormente la evolución fue tórpida y se realizó nefrectomía por déficit funcional severo.

En 2 casos $(0,04 \%)$ se produjo una rotura renal. El primero (Fig. 2) fue un paciente con severa ectasia renal previa por megauréter obstructivo izquierdo con litiasis en uréter terminal de $3,5 \mathrm{~cm}$ de diámetro máximo y tras tratamiento con lithoclast durante 60 minutos, que logró la fragmentación de 2/3 ó 3/4 del cálculo. Debido a un cuadro de dolor abdominal acompañado de fiebre, hematuria intensa y descenso de 16 puntos del hematocrito a las 32 horas se realizó una TC, la cual evidenció una colección líquida renal izquierda de gran tamaño por rotura del tracto urinario superior sin observarse colección periureteral distal. El caso se resolvió con medidas conservadoras (reposo absoluto y soporte hemodinámico) y posteriormente, una vez reabsorbido el extravasado, se realizó una ureterolitotomía del cálculo residual. El segundo paciente tenía una litiasis en uréter ilíaco derecho de $1,5 \mathrm{~cm}$, con ureterohidronefrosis moderada, que se trató con láser Candela ${ }^{\circledR}$ durante 32 minutos; a las 12 horas presentó un cuadro similar al del primer paciente, con caída de 17 puntos del hematocrito, y con los mismos hallazgos en la TC. En este caso no se consiguió estabilizar hemodinámica- 


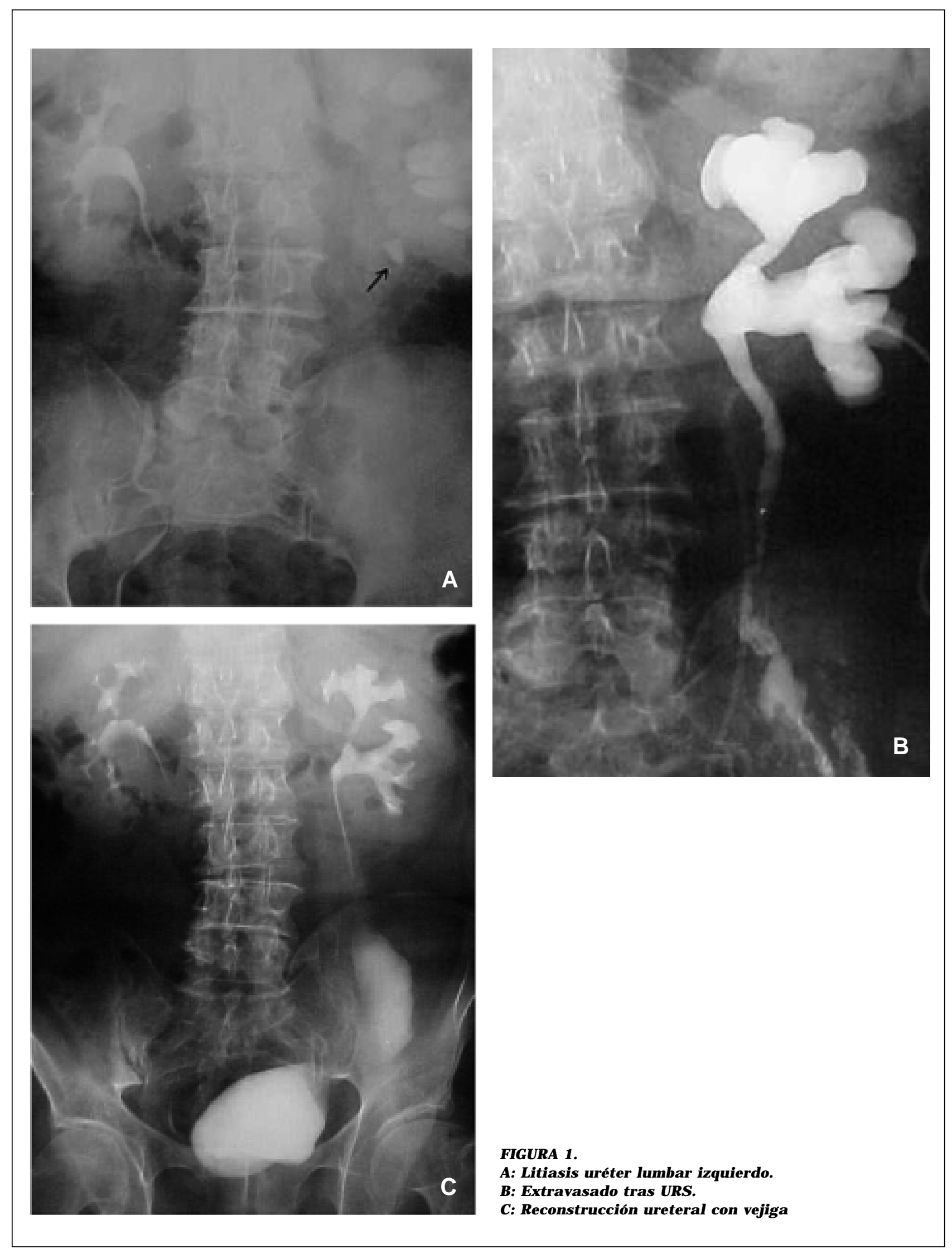



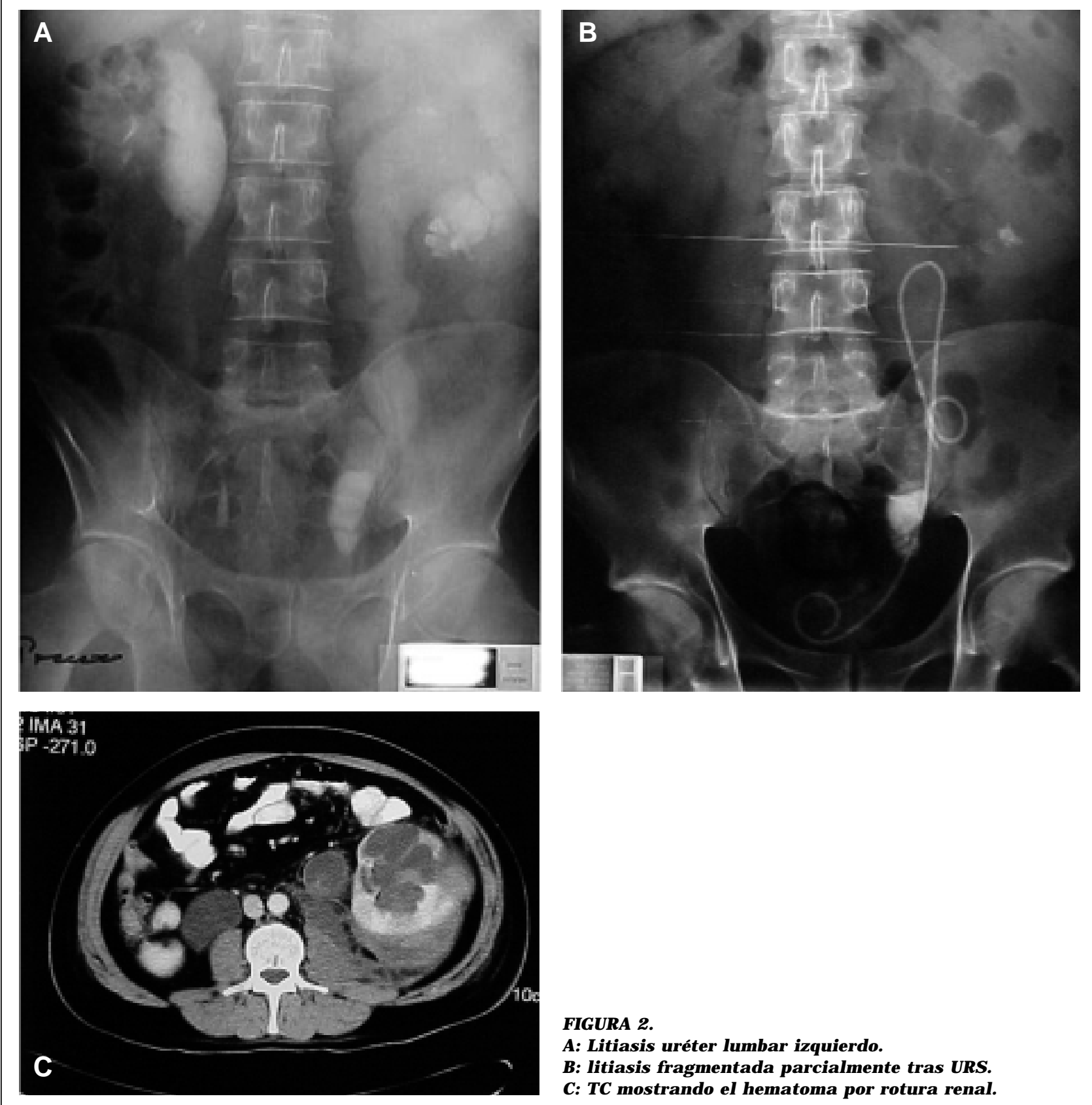

FIGURA 2.

A: Litiasis uréter lumbar izquierdo.

B: litiasis fragmentada parcialmente tras URS.

C: TC mostrando el hematoma por rotura renal.

mente al paciente con medidas conservadoras por lo que fue necesaria una nefrectomía inmediata.

En 19 pacientes $(0,4 \%)$ se produjo una extrusión litiásica paraureteral (Fig. 3), es decir la impactación de los cálculos que produce la salida de los mismos fuera de la mucosa ureteral quedando alojados en situación submucosa, en 5 hombres y 14 mujeres. El tamaño de estos cálcu- los fue de 4-13 mm (media de 7), 14 estaban localizados en el uréter ilíaco y el resto en el uréter lumbar. El diagnóstico de esta complicación fue mediante urografía intravenosa en 18 casos. En un paciente fue durante la URS ya que se produjo una perforación ureteral con extrusión de la litiasis, resolviéndose el cuadro con nefrostomía percutánea inmediata y posterior colocación de catéter doble $\mathrm{J}$ a las 4 semanas. Todos los casos 

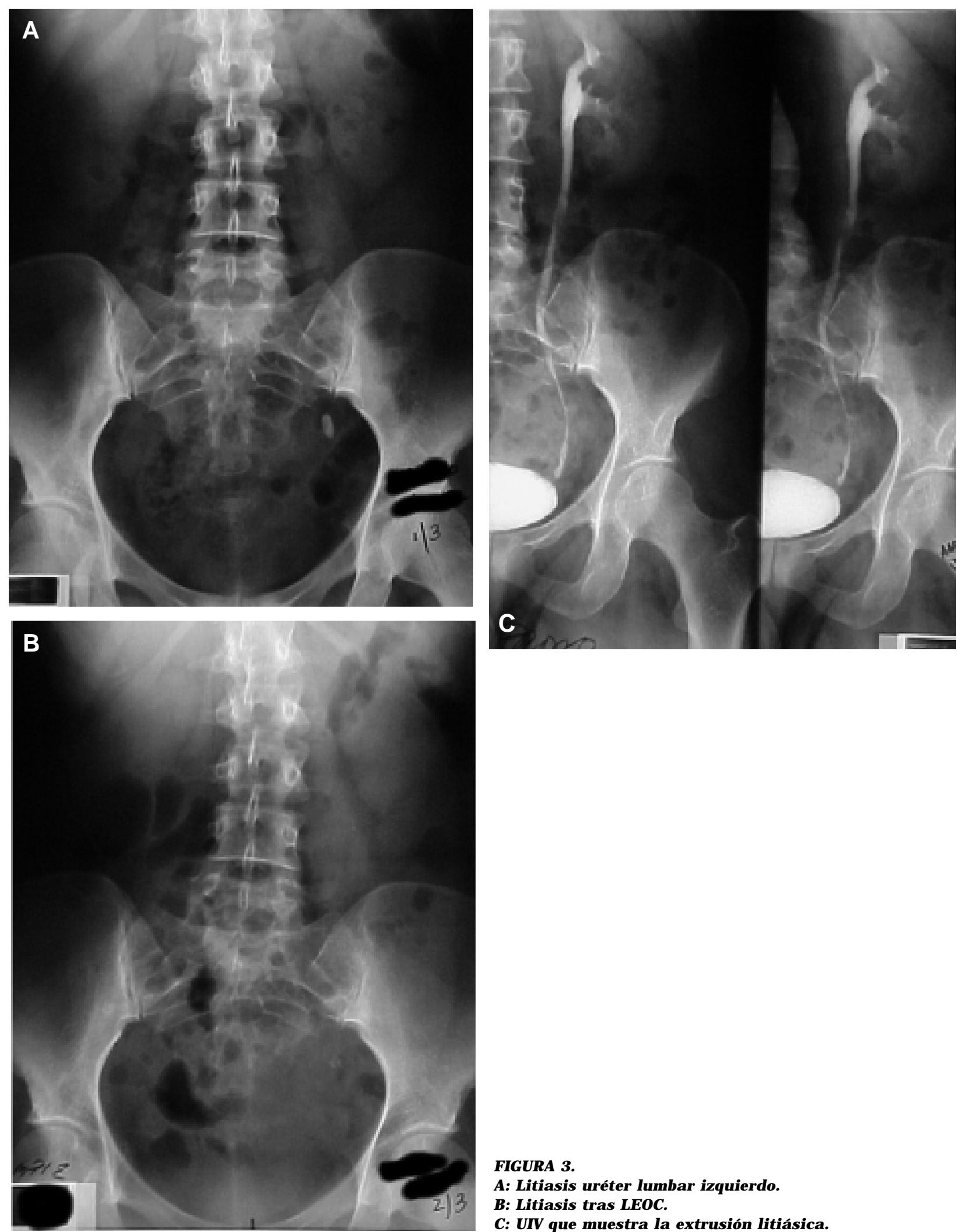

FIGURA 3.

A: Litiasis uréter lumbar izquierdo.

B: Litiasis tras LEOC.

C: UIV que muestra la extrusión litiásica. 
fueron resueltos con tratamiento conservador y tras un seguimiento medio de 85 meses (rango 74-101) la evolución ha sido satisfactoria, sin observarse extravasación urinaria, infecciones o estenosis secundaria por lo que no ha sido necesario extraer las litiasis extruidas en ningún caso.

Una de las complicaciones más severas de esta serie ha sido una fístula urétero-ilíaca $(0,02 \%)$ en un paciente de 44 años con litiasis en uréter pélvico izquierdo de $0,5 \mathrm{~cm}$ de diámetro máximo, que había sido tratada de manera infructuosa con LEOC. Se procedió a litofragmentación mediante URS y láser Candela ${ }^{\circledR}$ con anestesia raquídea. Tras 2.120 pulsos y con la litiasis prácticamente fragmentada, el paciente presenta un cuadro repentino de vómitos que provoca la flexión del tronco con el ureteroscopio insertado hasta uréter ilíaco. A consecuencia de este movimiento brusco se produjo inmediatamente un profuso sangrado intraureteral acompañado de un cuadro de hipotensión con gran inestabilidad hemodinámica (precisó trasfusión de 12 unidades de concentrado de hematíes y 2 unidades de plasma fresco congelado), por lo que se realizó laparotomía exploradora apreciando una lesión en la arteria hipogástrica izquierda y precisando una sutura arterial y colocación de catéter doble J. Posteriormente y tras la retirada del catéter el paciente presentó un urinoma por persistencia de la fístula ureteral que tras intento fallido de tratamiento percutáneo se resolvió finalmente con reimplante ureteral. La evolución posterior fue satisfactoria.

\section{DISCUSIÓN}

En la última década la URS se ha convertido en un importante instrumento para el diagnóstico y tratamiento de diferentes procesos renales y ureterales, sobre todo la litiasis. Sin embargo, es una técnica que no está exenta de complicaciones, ocasionalmente muy graves. Entre ellas destacan perforaciones ureterales, hemorragias e infecciones urinarias (9-20\%) $)^{1,2}$.

La aparición de ureteroscopios de bajo calibre, semirrígidos o flexibles y de los nuevos sistemas de litotricia adaptados a la endoscopia (láser, lithoclast) han hecho que esta técnica sea más eficaz y con menor morbilidad.
En muchos centros la URS se realiza con anestesia raquídea o epidural ${ }^{3}$ y en algunos casos tan sólo con anestesia local o sedación intravenosa ${ }^{4-7}$. La anestesia general tiene la ventaja de que al producir una parálisis muscular general evita las potenciales lesiones ureterales que podrían producirse si el paciente se mueve mientras se le está realizando la URS. Nosotros somos partidarios de la anestesia regional y si se puede de la sedación ya que, y pese a tener un caso de lesión de la arteria ilíaca secundario a un movimiento brusco del paciente durante la URS, creemos que en los casos en los que se pueda realizar y explicando bien al paciente que debe intentar no moverse o que si lo hace debe avisar antes, esta técnica anestésica ofrece mayores ventajas; así la presencia de dolor abdominal puede indicarnos la existencia de una perforación ureteral inadvertida durante la URS, finalizando de inmediato la exploración. En las mujeres la sedación permite realizar la URS sin necesidad de ingresar a la paciente, disminuyendo así las estancias hospitalarias y los costes económicos.

Desde la aparición de los miniscopios de bajo calibre no efectuamos una dilatación previa del meato. Siempre utilizamos una guía de seguridad y habitualmente una guía de trabajo para reducir el número de complicaciones.

Está ampliamente aceptado que el número de complicaciones de la URS disminuye con el uso de ureteroscopios flexibles y con la adquisición de experiencia por parte del urólogo ${ }^{8}$, si bien con el flexible la visión es peor y está limitado el uso del láser. En nuestro caso la mayoría de las complicaciones se produjeron durante los 3 primeros años de aprendizaje de cada uno de los miembros.

Compartimos la opinión de Borboroglo et al. ${ }^{9}$ de no dejar catéter doble $\mathrm{J}$ tras realizar URS no complicadas para el tratamiento de cálculos ureterales distales y siempre que se hayan extraído con pinzas el cálculo o la totalidad de los fragmentos. Desde 1999 esa es nuestra política, no colocamos doble $\mathrm{J}$ sistemáticamente con excepción de aquellos casos con edema intenso, perforación ureteral y extrusión de cálculos o restos litiásicos abundantes, sin haber observado ningún caso de obstrucción ni de estenosis ureteral en estos pacientes, evitando así el síndrome miccional irritativo, dolor en la fosa renal y riesgo de infección ligados al catéter. Pero ante la mínima 
duda lo mejor es dejar un catéter de doble $\mathrm{J}$, incluso con hilo para extraerlo sin necesidad de cistoscopia a las 48-72 horas.

El riesgo de perforación ureteral se incrementa cuando se tratan litiasis impactadas de larga evolución, siempre que el acceso sea dificultoso y cuanto mayor sea el tiempo quirúrgico ${ }^{10}$. En nuestra serie de 56 perforaciones, 38 (68\%) se produjeron tras tratamiento de litiasis mayores de $1 \mathrm{~cm}$ (media 1,5 ) y con duración mayor a 50 minutos (media 70). En los casos apreciados durante la URS finalizamos ésta inmediatamente tras colocar un catéter doble J. No hemos encontrado ningún caso de rotura renal tras URS descrito en la literatura. Nosotros aportamos 2 pacientes que presentaban los factores de riesgo de perforación ureteral descritos por Schuster et al. ${ }^{10}$ y que además tenían una ureterohidronefrosis bilateral severa, por lo que creemos que el mecanismo de rotura renal fue la hiperpresión mantenida en un riñón con cortical disminuida. Así recomendamos que en pacientes de estas características, especialmente con litiasis ureterales impactadas mayores de $2 \mathrm{~cm}$ y parénquima renal con cierto grado de atrofia, el tratamiento de elección debe ser la ureterolitotomía o bien la realización de URS previa colocación de nefrostomía percutánea que impida la sobrepresión.

La extrusión ureteral de pequeños fragmentos litiásicos suele pasar desapercibida durante la URS y se diagnostica en el control radiológico posterior (urografía iv). Es una complicación cuyo tratamiento debe ser conservador ya que el cálculo extruido no mantiene ningún contacto con la orina y por lo tanto no aumenta de tamaño. Tan sólo en las litiasis infectadas (estruvita) existe riesgo de absceso periureteral, incluso años después $^{11}$. En nuestra serie tras un seguimiento medio de 85 meses ningún paciente ha presentado esta complicación infectiva.

La avulsión ureteral es una complicación infrecuente de la URS, variando las series del $0,2-1 \%^{12,13}$. El diagnóstico suele ser inmediato al ver el uréter arrastrado a la vejiga mientras se está realizando la URS. El tratamiento es fundamentalmente quirúrgico y la técnica a realizar depende de la edad del paciente, función del riñón afecto, nivel y amplitud del defecto ureteral. En nuestra serie cada paciente fue tratado de modo individualizado.
En la revisión bibliográfica efectuada no hemos encontrado ningún caso de lesión arterial severa. En el que tuvimos la lesión se produjo por el movimiento brusco del paciente contra el ureterorrenoscopio introducido a nivel ilíaco. La intensa hematuria y la inestabilidad hemodinámica obligan a la laparotomía exploradora inmediata.

En conclusión la URS realizada cuidadosamente es una técnica eficaz y segura para el diagnóstico y tratamiento de las distintas afecciones del tracto urinario superior. Como en otros procedimientos, la URS también tiene una curva de aprendizaje por lo que recomendamos para disminuir el número de complicaciones la realización de ésta de una forma muy cuidadosa y el uso sistemático de una guía de seguridad y de una guía de trabajo para poder colocar un catéter doble $\mathrm{J}$ en caso de lesión ureteral

\section{REFERENCIAS}

1. SCHULTZ A, KRISTENSEN JK, BILDE T, ELDRUP J.: Ureteroscopy: results and complications. J Urol 1987; 137: 865-866.

2. BLUTE ML, SEGURA JW, PATTERSON DE.: Ureteroscopy. J Urol 1988; 139: 510-512.

3. JENKINS AD.: ESWL: Distal ureteral stone management. Urology 1993; 42: 353-355.

4. SCHOW DA, JACKSON TL, SAMSON JM, HIGHTOWER SA JOHNSON DL.: Use of intravenous alfentanil-midazolam anesthesia for sedation during brief endourologic procedures. $J$ Endourol 1994; 8: 33-36.

5. RITTENBERG MH, ELLIS DJ, BAGLEY DH.: Ureteroscopy under local anesthesia. Urology 1987; 30: 475-478.

6. VOGELI TA, MELLIN HE, HOPF B, ACKERMAN R.: Ureteroscopy under local anesthesia with and without intravenous analgesia. Br J Urol 1993; 72: 161-164.

7. HOSKING DH, BARD RJ.: Ureteroscopy under intravenous sedation for treatment of distal ureteral calculi: a safe and effective alternative to shock wave lithotripsy. J Urol 1996; 156: 899-901.

8. HARMON WJ, SERSHON PD, BLUTE ML, PATTERSON DE, SEGURA JW.: Ureteroscopy: current practice and long term complications. J Urol 1997; 157: 28-32.

9. BORBOROGLU PG, AMLING C L, SCHENKMAN NS et al.: Ureteral stenting after ureteroscopy for distal ureteral calculi: a multi-institutional prospective randomized controlled study assessing pain, outcomes and complications. J Urol 2001; 166: 1.651-1.657.

10. SCHUSTER TG, HOLLENBEG BK, FAERBER GJ, WOLF JS.: Complications of ureteroscopy: analysis of predictive factors. $J$ Urol 2001; 166: 538-540.

11. LANG EK.: Percutaneous nephrolithotomy and lithotripsy: multiinstitutional survey of complications. Radiology 1987; 162: 25-30.

12. PUPPO P, RICCIOTTI G, BOZZO W, INTROINI C.: Primary endoscopic treatment of ureteric calculi. Eur Urol 1999; 36: 48-52.

13. OKTAY B, YAVASÇAOGLU I, SIMSEK Ü, ÖYZURT M.: Intracorporeal pneumatic lithotripsy for ureteral and vesical calculi. Scand J Urol Nephrol 1997; 31: 333-336.

Dr. J.M. Alapont Alacreu

Servicio de Urología. Hospital Universitario La Fe

Avda. Campanar 21 - 46009 Valencia

(Trabajo recibido el 17 enero 2003) 